\title{
Vía aérea difícil
}

\author{
Dr. Pablo Bertrand', Dra. María Angélica Oyarzún² \\ 1 Profesor Asociado, Escuela de Medicina. Pontificia Universidad Católica de Chile. \\ 2 Residente de Enfermedades Respiratorias del Niño. Pontificia Universidad Católica de Chile.
}

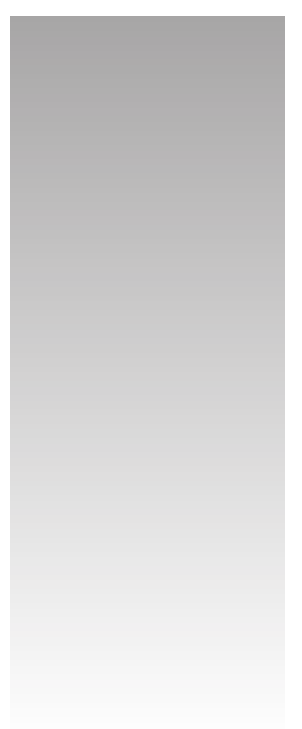

\section{DIFFICULT AIRWAY}

Difficult airway is a life-threatening situation which compromises the permeability of the upper airway and thus adequate ventilation and oxygenation. Multiple factors, acute and chronic such as: infectious, neoplastic and trauma have been associated with critical airway. Morbidity and mortality related to a difficult airway management remains as a significant problem in children, so is essential for the pediatric health team to be trained to recognize and anticipate situations that in clinical practice might determine a critical airway. The aim of this review is to provide concepts and guidance to assess patients with potentially difficult airway.

Key words: Difficult airway; airway management, acquired upper airway obstruction, craniofacial anomalies.

\section{RESUMEN}

Una vía aérea difícil condiciona una situación con riesgo vital, ya que pone en peligro la permeabilidad de la vía aérea superior y con esto la capacidad de mantener una adecuada ventilación y oxigenación. Múltiples factores, tanto agudos como crónicos, entre ellos factores anatómicos propios del niño/a, complicaciones infecciosas, neoplásicas y/o traumáticas se han asociado con una vía aérea crítica. La morbilidad y mortalidad asociada al manejo inadecuado de esta condición continua siendo un problema significativo en la edad pediátrica; siendo fundamental que el equipo de salud se encuentre entrenado en reconocer y anticipar situaciones que en la práctica clínica podrían asociarse con una vía aérea difícil o crítica. El objetivo de la presente revisión es otorgar conceptos y una orientación en el enfrentamiento de los pacientes con una vía aérea potencialmente difícil.

Palabras clave: Obstrucción vía aérea superior, vía aérea difícil, manejo vía aérea, anormalidades craneofaciales.

\section{INTRODUCCIÓN}

La permeabilidad de la vía aérea superior es fundamental para mantener una adecuada ventilación y oxigenación, de modo que cualquier dificultad para mantenerla despejada condiciona una situación de riesgo vital para el niño, lo que se denomina vía aérea difícil (VAD). Múltiples factores, tanto agudos como crónicos, pueden determinar el escenario de una VAD, como factores anatómicos normales del desarrollo de un niño, complicaciones infecciosas, neoplásicas y/o traumáticas. La morbilidad y mortalidad asociada al manejo inadecuado de esta condición ha mostrado un descenso durante el último tiempo, pero continua siendo un problema significativo en la población pediátrica y es por esta razón que el equipo de salud debe estar entrenado en reconocer y anticipar situaciones que en la práctica clínica podrían asociarse con una vía aérea difícil o también conocida como vía aérea crítica.

\section{DEFINICIÓN}

No existe consenso para una definición única de VAD. En el contexto de la anestesia se define como: "aquella situación en

Correspondencia: Dr. Pablo Bertrand Lira $85-5^{\circ}$ piso Laboratorio de Respiratorio Pediátrico. Santiago, Chile.

E-mail: Bertrand@med.puc.cl

ISSN 07|8-332| Derechos reservados. que un anestesista entrenado presenta dificultad en la ventilación con máscara, intubación traqueal o ambas aunque se usen técnicas óptimas"(I); siendo resultado de una compleja interacción entre condiciones clínicas del paciente, disponibilidad de equipamiento y habilidades del operador ${ }^{(2)}$. En los niños, la vía aérea difícil, ya sea congénita o adquirida puede presentarse de forma aguda, potencialmente mortal o bien de forma crónica y progresiva, de manera que conduce a insuficiencia respiratoria crónica. Son tres situaciones las que resumen una VAD: dificultad en obtener un buen sello con la ventilación con máscara y bolsa, dificultad en visualizar las cuerdas vocales para introducir un tubo endotraqueal y dificultad en lograr ventilar por obstrucción distal al tubo endotraqueal. La situación más temida denominada "no intubable, no ventilable" afortunadamente es poco frecuente en niños ${ }^{(3)}$.

\section{ANATOMÍA}

Existen diferencias relevantes entre la vía aérea pediátrica y la del adulto, lo que puede constituir una dificultad en su manejo. La vía aérea del niño posee un diámetro menor, sus narinas son pequeñas y la lengua proporcionalmente más grande. Además presenta un cuello corto que localiza la glotis en posición más cefálica a nivel de C3-C4 y la epiglotis tiene forma de omega, más larga, laxa y con frecuencia colapsable por inmadurez cartilaginosa. En la edad prescolar el niño presenta amígdalas y adenoides que han crecido para alcanzar 
su máximo tamaño entre los cuatro y siete años de edad. La laringe tiene forma de embudo en menores de 8 años y su zona más estrecha, está a nivel del anillo cricoides, donde la submucosa está ricamente vascularizada siendo susceptible a inflamación y edema. Esto condiciona que la presencia de edema de la mucosa de $1 \mathrm{~mm}$ disminuya el calibre de su lumen en $75 \%$, mientras que en el adulto sólo se reduce un $20 \%$. Por otro lado, el tamaño craneano mayor y occipucio prominente resulta en una posición subóptima para el abordaje de la vía aérea al momento de realizar una laringoscopia o intubación endotraqueal.

\section{SÍNDROMES GENÉTICOS}

Muchos síndromes genéticos condicionan la presencia de una vía aérea difícil, sobre todo en la edad pediátrica cuando las proporciones craneofaciales son críticas en determinar obstrucción de la vía aérea superior. La permeabilidad de la vía aérea se puede comprometer por alteraciones craneofaciales que incluyen craneosinostosis o cierre precoz de las suturas craneales, lo que limita el crecimiento y distorsiona el cráneo, acortamiento de las cavidades nasales, del paladar y maxilar, hipoplasia facial, prognatismo mandibular, puente nasal deprimido, arco palatino alto, mala oclusión dental, paladar hendido y úvula bífida como ocurre en los Síndromes de Apert, Crouzon y Pfeiffer entre otros ${ }^{(4)}$. Por otro lado, malformaciones del arco branquial como las disostósis mandibulofaciales pueden asociarse con micro o macrostomía y micrognatia, un ejemplo de ello es el síndrome de Treacher-Collins que presenta hipoplasia de huesos y músculos faciales, hipoplasia faríngea, mala oclusión dental y arcada palatina alta. Pacientes con síndrome de Goldenhar pueden presentar hipoplasia facial malar, maxilar, temporal o mandibular, hipoplasia de músculos de la masticación, deformaciones de la lengua y paladar, anomalías vertebrales cervicales, labio y paladar hendido y alteraciones laríngeas ${ }^{(5)}$. Otros síndromes misceláneos incluyen algunas condiciones particularmente frecuentes; el síndrome de Down que presenta hipotonía de la faringe y gran tamaño de la lengua lo que condiciona obstrucción de la vía aérea significativa que puede llegar a ser crítica ante procedimientos que requieran de sedación profunda o anestesia. Además se ha demostrado que la vía aérea del niño con síndrome de Down es más pequeña que la de un niño normal de la misma edad. La secuencia de Pierre Robin es otra entidad que constituye un desafío en la vía aérea puesto que condiciona una obstrucción significativa de la región faríngea. Estos niños presentan mandíbula pequeña, posición baja de la laringe y paladar hendido, lo que resulta en obstrucción crónica sobre todo durante los primeros años de vida. Niños con síndrome de CHARGE (Coloboma, Heart- cardiopatía, Atresia de coanas, Retardo de crecimiento y/o desarrollo, anomalías Genitales y/o urinarias y Eyes - anormalidades oculares y sordera) con relativa frecuencia es asociada a una VAD y por último en el síndrome de Beckwith-Wiedemann la severa macroglosia y obstrucción de la vía aérea secundaria que presentan justifica en muchas casos necesidad de traqueostomía. De todos estos síndromes genéticos, aquellos con creaneosinostosis y disostosis mandibulofacial presentan mayor frecuencia de vía aérea crítica con alto riesgo de requerir traqueostomía (alrededor del $40 \%$ ) como solución durante los primeros años de vida al ser comparada con el grupo de microsomías hemifaciales (alrededor del 20\%) y las otras condiciones nombradas que presentan menor riesgo( ${ }^{(6)}$.

\section{CONDICIONES CRÓNICAS}

Múltiples condiciones pueden determinar una VAD en forma crónica, la mayoría en forma progresiva y lenta o cuando la obstrucción ha alcanzado un punto crítico desencadenada por una situación aguda (Tabla I). Un buen ejemplo de esta situación es la laringomalacia, anomalía laríngea congénita caracterizada por colapso de las estructuras laríngeas, lo que puede condicionar obstrucción de vía aérea superior (OVAS) de severidad variable. El niño suele presentarse durante los primeros meses de vida con estridor que se exacerba en situaciones de esfuerzo como agitación, alimentación, llanto y muchas veces por la posición supina. En la mayoría de los casos esta situación no amerita ninguna intervención; sin embargo, algunos niños pueden presentar dificultad respiratoria, crisis de cianosis, apneas, dificultad para alimentarse y falta de incremento ponderal. Ante estos signos de severidad es fundamental anticipar una vía aérea crítica en situaciones de vulnerabilidad como infecciones, sedación y anestesia ${ }^{(7)}$. Otra condición frecuente que puede generar vía aérea crítica es la estenosis subglótica, la mayoría de las veces adquirida como consecuencia de necrosis de la mucosa cricoidea y/o pericondritis de la región subglótica secundarias a intubación

\section{Tabla I. Condiciones crónicas asociadas con VAD}

\section{Síndromes genéticos}

I. Apert, Crouzon, Pfeiffer

2. Treacher-Collins, Nager, microsomía hemifacial

3. Goldenhar, CHARGE

4. Sd Down, Secuencia Pierre Robin

5. Sd Klippel-Feil, Sd Beckwith- Wideman

Estructurales

I. Macroglosia (Sd. Berckwith-Wideman, hipotiroidismo, Sd. Down)

2. Hipertrofia adenotonsilar

3. Obesidad

4. Laringo / traqueomalacia

5. Estenosis subglótica (congénita y adquirida)

6. Parálisis cuerdas vocales (congénita y adquirida)

7. Compresión extrinseca (anillos vasculares, cardiomegalias, neoplasias mediastinicas, enfisema lobar congénito)

Tumorales-Neoplásicas

I. Higroma quístico

2. Teratoma quístico

3. Papilomas

4. Hemangiomas

5. Linfangiomas

6. Quistes, neurofibromas 
endotraqueal prolongada, traumática o reiterada. La sintomatología dependerá del grado de obstrucción y habitualmente se utiliza la clasificación de visualización endoscópica de Myer$\mathrm{O}^{\prime}$ Connor-Cotton (Tabla 2) para establecer severidad. Estos niños desarrollan estridor progresivo bifásico posterior a la noxa que se asocia a una manifiesta dificultad respiratoria con polipnea, tiraje, retracción de partes blandas, eventualmente cianosis y apneas. Nuevamente en esta situación es vital anticipar una vía aérea crítica ante escenarios de vulnerabilidad. Muchas condiciones congénitas o adquiridas durante los primeros años de vida ameritan anticipar una VAD aun cuando se presentan de forma crónica.

La parálisis de cuerda vocal unilateral puede condicionar obstrucción parcial de la vía aérea y se presenta como consecuencia de injuria ante un parto distócico o cirugía de mediastino y/o cervical. Suele ser de manejo conservador aunque distintas situaciones pueden vulnerar la permeabilidad a este nivel. La parálisis de cuerda bilateral está asociada a daño central congénito como ocurre en la malformación de Arnold-Chiari y se presenta con estridor, voz débil y dificultad de la deglución. En esta situación la permeabilidad de la vía aérea habitualmente está comprometida de forma severa.

\section{CONDICIONES AGUDAS}

Una variedad de condiciones agudas pueden asociarse con obstrucción de la vía aérea superior y condicionar una vía aérea crítica, entre ellas causas infecciosas, traumáticas o aspiración de cuerpo extraño (Tabla 3). El área subglótica corresponde a la zona más estrecha de la vía aérea del niño y es particularmente vulnerable porque el cartílago cricoides se dispone como un anillo completo que facilita la obstrucción aguda que puede progresar rápidamente hacia el colapso completo de la vía aérea.

La causa más común de obstrucción aguda de la vía aérea superior es el croup o laringitis obstructiva viral, cuadro caracterizado por voz ronca, tos perruna y estridor que ocurre como consecuencia de la infección por virus parainfluenza como principal agente. Se presenta en niños de 6 meses a 6 años de edad, momento del desarrollo en que los factores anatómicos predisponen al paciente a la obstrucción crítica

\section{Tabla 3. Condiciones agudas asociadas a VAD}

\begin{tabular}{|ll|}
\hline Causas Infecciosas & Traumáticas \\
- Coup o laringitis obstrucctiva & - Parálisis de cuerdas vocales \\
- Croup espasmódico & - Daño térmico \\
- Epiglotitis & - Lesiones caústicas \\
- Traqueitis bacteriana & - Injuria post procedimientos \\
- Mononucleosis & - Estenosis subglótica \\
- Absceso retrofaríngeo. & \\
- Absceso periamigdaliano & \\
Aspiración de cuerpo extraño & Angioedema hereditario \\
& (anafilaxia) \\
Neoplásicas & Disfunción de cuerdas vocales \\
\hline
\end{tabular}

*Adaptado Hammer J. Acquired upper airway obstruction. Paediatric Respiratory Reviews 2004; 5: 25-33.
Tabla 2. Clasificación Myer- O'Connor-Cotton. Severidad de estenosis subglótica según visualización
endoscópica

\begin{tabular}{|ll|}
\hline Grado I & $50 \%$ o menos de obstrucción del lumen de VA \\
Grado 2 & Obstrucción entre 5 I y $70 \%$ del lumen de VA \\
Grado 3 & $\begin{array}{l}\text { Obstrucción mayor al } 70 \% \text {, con visualización del lumen } \\
\text { de VA }\end{array}$ \\
Grado 4 & No se observa lumen de VA \\
\hline
\end{tabular}

en el punto de mayor estrechez correspondiente a la región subglótica. Para clasificar su severidad se propone usar el puntaje de Westley que valora los signos de dificultad respiratoria que pueden ayudar a anticipar una vía aérea crítica, como la presencia de estridor espiratorio, respiración laboriosa, polipnea, aleteo nasal, pulso paradojal y desaturación los que constituyen factores predictores de falla respiratoria. El croup espasmódico se presenta de forma muy similar al croup viral pero de manera recurrente. Suele presentarse sin pródromo infeccioso y en el contexto de un niño con alergia y asma. Otras infecciones de la vía aérea que pueden condicionar una obstrucción significativa son aquellas que comprometen distintas estructuras blandas que impiden la permeabilidad normal de la vía aérea como absceso retrofarigeo, absceso periamigdalino y la mononucleosis infecciosa o aquellas que son parte del diagnóstico diferencial del croup viral: traqueítis bacteriana y epiglotitis. Este último cuadro es muy infrecuente hoy en día, pero de temida severidad como condicionante de vía aérea crítica. Las características 4D que describen al paciente: disfagia, disfonía, disnea y salivación (drooling en inglés) son síntomas que deben alertar al clínico de una situación de rápida progresión y potencialmente fatal( ${ }^{(7)}$.

La reacción de tipo anafiláctico conocida como angioedema hereditario constituye una causa poco común pero extremadamente grave de vía aérea crítica. Se presenta en general en pacientes durante la edad de la pubertad y puede ser recurrente. Está dentro de las condiciones que definen la anafilaxis y hoy se ha establecido su relación con la deficiencia del inhibidor de estereasa $\mathrm{Cl}$. Mención especial merece la obstrucción de la vía aérea por cuerpo extraño, accidente relativamente frecuente en niños y principal causa de muerte accidental en menores de 5 años. Los síntomas dependen de su naturaleza, localización y grado de obstrucción de la vía aérea; la mayoría de las veces la aspiración de un cuerpo extraño migra hasta primera o segunda generación bronquial, pero la impactación en la laringe determina una vía aérea crítica y una emergencia médica. El diagnóstico precoz y el tratamiento inmediato son fundamentales ya que la obstrucción puede ser de curso rápido y mortal.

\section{EVALUACIÓN}

La anticipación de una vía aérea crítica supone realizar una historia clínica detallada que permita evaluar características anatómicas y fisiológicas de riesgo, además del lugar más vulnerable de obstrucción. Estos factores pueden ser congénitos, traumáticos, inflamatorios, neoplásicos y metabólicos , 
Tabla 4. Clasificación de Mallampati

\begin{tabular}{|cl|}
\hline & Estructuras visibles \\
\hline Clase I & Paladar blando, úvula y pilares amigdalinos \\
\hline Clase li & Paladar blando y úvula \\
\hline Clase lii & Paladar blando y base de la úvula \\
\hline Clase Iv & Imposibilidad para ver el paladar blando \\
\hline
\end{tabular}

pudiendo clasificarlos según el sitio anatómico de obstrucción. En la historia del paciente antecedentes clínicos como presencia de ronquido, estridor, respiración ruidosa, somnolencia, dificultad respiratoria con la alimentación o apneas del sueño sugieren una vía aérea superior lábil ante cualquier intervención. Información relativa a morbilidad previa como croup recurrente, absceso amigdaliano, cirugías previas de cabeza y cuello, radioterapia en cualquier segmento de la vía aérea y tumores pueden condicionar una VAD. Por último condiciones asociadas que pueden determinar un mayor riesgo ante un procedimiento como la presencia de aspiración pulmonar crónica por daño neurológico o reflujo gastroesofágico deben ser tomados en cuenta por la abundante secreción que puede presentar el niño y la dificultad que esto trae en el éxito de cualquier procedimiento.

La exploración física completa de cabeza, cuello y columna cervical debe ser exhaustiva y metódica para anticipar puntos de obstrucción. Se debe observar permeabilidad de las narinas, apertura oral, arcada dental, movilidad temporomandibular y cervical. El aspecto general del niño es un indicador confiable de severidad, de modo que la presencia de polipnea, uso de musculatura accesoria, cianosis y estridor en reposo sugieren una potencial VAD.

Existen mediciones clínicas utilizadas en población adulta, sin embargo, no han sido validadas en niños y son difíciles de aplicar en pacientes que no colaboran. Entre las más utilizadas están la clasificación de Mallampati (Tabla 4) que valora la visualización de las estructuras anatómicas faríngeas con el paciente sentado con la boca completamente abierta y la distancia tiroidea mentoniana que valora distancia existente entre la escotadura superior del cartílago tiroides y el borde inferior del mentón en posición sentada con la cabeza extendida y la boca cerrada. Ambas poseen baja sensibilidad y especificidad para pronóstico de vía aérea crítica.

Estudios adicionales de laboratorio pueden ser de gran utilidad para anticipar una VAD tanto en condiciones de evolución aguda o crónica. En forma general, los gases arteriales podrían mostrar la presencia de hipoxemia y/o hipercapnia como manifestación de hipoventilación pero su utilidad es baja puesto que estas alteraciones suelen ser tardías.

El estudio de función pulmonar con curvas flujos/volumen puede demostrar obstrucción de vía aérea fija o variable, extra o intratorácica y orientar la necesidad de estudios invasivos, aunque la utilidad de estas mediciones está limitada a su realización en niños que colaboren y que no presenten obstrucción significativa en el momento de la prueba.

Los estudios de imágenes, desde radiografía tórax en sus proyecciones anteroposterior y lateral, la tomografía axial computada o resonancia nuclear magnética cervical y de tórax tienen como objetivo definir con mayor precisión el carácter y extensión de una determinada lesión, como en el caso de absceso retrofaringeo, periamigdaliano, tumores de vía aérea, linfangiomas o higromas cervicales y obstrucción traqueal por compresión extrínseca entre otros. La utilidad varía de acuerdo a la condición que se considera y generalmente se transforma en un complemento de la evaluación endoscópica.

La visualización de la vía aérea se puede realizar por laringoscopia directa, broncoscopía rígida y flexible. Todos estos procedimientos permiten analizar de forma directa la permeabilidad de la vía aérea y estimar el riesgo de cada paciente en desarrollar una VDA. Los resultados son dependientes directamente de la situación clínica y de la destreza del operador. La broncoscopía flexible tiene como ventajas evitar anestesia general, baja incidencia de complicaciones y favorece el estudio dinámico de la vía aérea hasta la tercera y cuarta generación bronquial, pero como complemento la broncoscopía rígida permite evaluar con mayor precisión la patología de cuerdas vocales, glotis posterior, subglotis y tráquea además de complementar con técnicas invasivas la certeza diagnóstica a través de la toma de muestras.

\section{MANEJO}

El enfrentamiento de un paciente con vía aérea difícil debe considerar el nivel en que ocurre la obstrucción, la forma de presentación (aguda o crónica) y el escenario clínico en que se presenta, sea en el ámbito anestésico o fuera de este. Para cualquier condición el enfoque de una situación compleja debe ser multidisciplinaria con la participación de intensivistas, especialistas en endoscopía y equipo quirúrgico. Independiente de la etiología de la obstrucción, el objetivo principal de su manejo debe ser mantener la permeabilidad de la vía aérea. Se debe considerar que la principal diferencia entre el niño y el adulto no radica en algún elemento anatómico crítico del niño sino en la rapidez con que este paciente desarrolla hipoxemia significativa.

El tratamiento de la vía aérea crítica comienza con recuperar o mantener la permeabilidad de esta a través de la ventilación con máscara y bolsa. La habilidad para realizar estas maniobras es fundamental en el manejo de la vía aérea. Los elementos para realizar ventilación de esta forma están disponibles en todos los niveles de complejidad de atención médica y debiera ser dominada por todo pediatra que trabaja en unidad de emergencia, hospitalizado o sala de procedimientos y anestesia. Muchas veces el problema para lograr una adecuada ventilación consiste en aplicar un buen sello en los bordes de la máscara. Para esto es indispensable posicionar de forma correcta la cabeza y traccionar la mandíbula para favorecer una ventilación gentil y eficiente. Recientemente se ha validado en muchos escenarios el uso de máscara laríngea para la mantención de la permeabilidad de la vía aérea; su manipulación es simple y el resultado es generalmente óptimo. La única dificultad en la instalación puede ser el desplazamiento de la epiglotis hacia distal con la resultante obstrucción de la vía aérea. El tercer paso y más invasivo consiste en realizar intubación endotraqueal bajo visualización directa 
con laringoscopio o asistida con fibrobroncoscopia flexible. Este procedimiento debe ser realizado por el operador más experto cuando se ha anticipado de forma correcta una VAD.

En situaciones en las que la vía aérea no constituye una emergencia inmediata la aproximación terapéutica va a depender de la causa que valora. En forma general, si la obstrucción aguda ocurre a nivel faríngeo, por ejemplo en abscesos periamigdalinos el tratamiento puede considerar el apoyo de CPAP, máscara laríngea o bien intubación endotraqueal, lo que dependerá de la estabilidad del paciente y disponibilidad de recursos. Ante obstrucción faríngea crónica, como ocurre en pacientes con enfermedades neuromusculares y/o niños con síndrome de Down se puede considerar el uso de tubos nasofaríngeos, apoyo con CPAP o traqueostomía si la situación lo amerita ${ }^{(8)}$. Algo similar ocurre en niños con síndrome de Beckwith-Wiedemann y secuencia Pierre-Robin; sin embargo, en estos pacientes es posible comenzar con posicionamiento, placas palatinas de ortodoncia y flujo nasal con cánulas nasofaringeas durante las primeras semanas de la vida disminuyendo la necesidad de traqueostomía, mientras se pueda realizar resolución quirúrgica con distracción ósea ${ }^{(9,10)}$.

Por otro lado, cuando la obstrucción aguda ocurre a nivel laríngeo supraglótico, como en la epiglotitis, el abordaje terapéutico está bastante claro, se debe administrar oxígeno, evitar procedimientos invasivos hasta que el paciente puede ser anestesiado con anestésicos inhalados, ventilado con máscara y luego proceder con acceso intravenosa establecido a intubación endotraqueal por el personal más calificado, ya que suele ser difícil.

En laringomalacia, el manejo habitualmente es conservador y sólo se debe plantear uso de traqueostomía ante signos de gravedad como apneas, cianosis, dificultad respiratoria o falta de progreso ponderal. Otra opción quirúrgica es la epigloplastía, que consiste en resecar partes de la epiglotis y pliegues ariepiglóticos superiores, lo que en general debiera ser hecho unilateralmente por el riesgo de estenosis cicatricial( ${ }^{(1)}$. En el caso de obstrucción por parálisis cordal unilateral, el niño suele lograr compensar el déficit y el manejo tiende a ser conservador, pero en el caso de parálisis bilateral es muy difícil evitar la traqueostomia por el alto riesgo de obstrucción severa ${ }^{(I)}$.

A nivel subglótico, el croup es la causa más común de obstrucción aguda y su tratamiento se encuentra bien estandarizado, logrando con el uso de corticoides sistémicos y nebulizaciones de adrenalina, el manejo adecuado de la mayoría de los pacientes, limitando sólo a los casos más severos el manejo invasivo de la vía aérea ${ }^{(12,13)}$. Por otro lado, la estenosis subglótica ya sea secundaria a inflamación/infección de la vía aérea, a intubación endotraqueal, o por la presencia de lesiones quísticas y/o hemangiomas habitualmente requieren como parte de su manejo el uso de traqueostomía, mientras se logra el manejo intervencional/quirúrgico de la patología de base $\mathrm{e}^{(14)}$.

\section{CONCLUSIONES}

El enfrentamiento de un paciente con vía aérea difícil es una situación clínica presente en la práctica pediátrica. El conocimiento y entrenamiento en condiciones conocidas por su asociación con vía aérea difícil es indispensable para anticipar y tratar la permeabilidad de la vía aérea. El manejo involucra la colaboración de equipos multidisciplinarios orientados a cuidado integral e individualizado de cada uno de estos pacientes. La principal diferencia entre el niño y el adulto radica en la rapidez en desarrollar hipoxemia en el primero con las conocidas consecuencias. La maniobra más importante en el manejo de la vía aérea es la ventilación con máscara y bolsa.

\section{REFERENCIAS}

1. Practice guidelines for management of the difficult airway: an updated report by the American Society of Anesthesiologists Task Force on Management of the Difficult Airway. Anesthesiology 2003; 98: 1269-77.

2. Andreu E, Schmucker E, Drudis R, Farre M, Franco T, Monclus E, et al. Algoritmo de la vía aérea difícil en pediatría. Rev Esp Anestesiol Reanim. Mayo 20 I ; 58: 304-II

3. Craig Sims, Britta von Unger The normal and the challenging pediatric airway. Pediatric Anaesth 20 I2; 22: 521-6.

4. Xue FS, Zhang YM, Liao X, Liu JH. Airway management for pediatric patients with difficult airways due to craniofacial abnormalities. Paediatr Anaesth 2009; 19: 701-2

5. Vener DF, Lerman J. Vías respiratorias pediátricas y síndromes relacionados con ellas. Rev Col Anest 1998; 26: 317.

6. Sculerati N, Gottlieb MD, MS Zimbler, McCarthy JG. Airway Managment in Children with mayor Craniofacial Anomalies. Laringoscope 1998; 108: 1806-12.

7. Hammer J. Acquired upper airway obstruction. Rev Respir Paediatr 2004; 5: 25-33.

8. Marcus CL, Keens TG, Bautista DB, Von Pechmann WS, Ward SLD. Obstructive sleep apnea in children with Down syndrome. Pediatrics 1991; 88: 132-9.

9. Rimell FL, Shapiro AM, Shoemaker DL, Kenna MA. Head and neck manifestations of Beckwith Wiedemann syndrome. Otolaryngol Head Neck Surg 1995; 1 I 3: 262-5.

10. Tomaski SM, Zalzal GH, Saal HM. Airway obstruction in the Pierre Robin sequence. Laryngoscope 1995; 105: I | |-4.

I I. Zalzal GH, Anon JB, Cotton RT. Epiglottoplasty for the treatment of laryngomalacia. Ann Otol Rhinol Laryngol 1987; 96: 72-6.

12. Newman KB, Mason UG 3rd, Schmaling KB. Clinical features of vocal cord dysfunction. Am J Respir Crit Care Med 1995; 152: 1382-6.

13. Stannard W, O'Callaghan C. Management of croup. Paediatr Drugs 2002; 4: 23I-40.

14. Nicolai T. Therapeutic concepts in upper airway obstruction. Rev Respir Paediatr 2004; 5: 34-9. 